\title{
Secuelas neurológicas del maltrato infantil. Revisión bibliográfica
}

\author{
J. Giménez-Pando; E. Pérez-Arjona*; M. Dujovny* y F.G. Díaz*
}

Servicio de Neurocirugía. Unidad de Neurocirugía infantil. Hospital Virgen del Rocío. Sevilla. España. *Departamento de Neurocirugía. Wayne State University. Detroit (MI). USA.

\section{Resumen}

El maltrato infantil es un problema de gran importancia tanto social como médico, y sus consecuencias en muchas ocasiones son irreparables. Realizamos una revisión bibliográfica con enfoque neuroquirúrgico, y analizamos con detalle las lesiones que se producen en el sistema nervioso central, estudiando su fisiopatología, las secuelas neurológicas que producen y su implicación en el tratamiento rehabilitador y el futuro de los niños.

PALABRAS CLAVE: Hemiplejia. Maltrato infantil. Secuelas. Trabajo social. Traumatismo craneoencefálico.

Neurolical sequels of chlid abuse. Literature review

\section{Summary}

Child abuse is both socially and medically troublesome and many times produces permanent consequences. A review of the literature is done from a neurosurgical standpoint, and the lesions produced at the Central Nervous System are evaluated in detail, including their physiopathology, neurological sequels and implications for rehabilitation treatment and the child's future life.

KEY WORDS: Child abuse. Head injury. Hemiplegia. Sequels. Social Work

\section{Introducción}

El maltrato infantil es un problema de gran importancia tanto social como médico. Los pediatras, neurólogos, psiquiatras, neurocirujanos, trabajadores sociales, especialistas en leyes y diversas agencias gubernamentales, entre otros, han descrito previamente el síndrome de maltrato infantil ${ }^{19}$. Sin embargo, los artículos neuroquirúrgicos rara vez se detienen a analizar en profundidad dicho problema.

Recibido; 3-04-06. Aceptado: 10-05-06
El objetivo de la presente revisión bibliográfica es analizar las secuelas neurológicas que se producen en los niños que sufren maltrato, con el fin de proporcionar a los médicos una mejor comprensión del problema y de sus consecuencias.

\section{Materiales y métodos}

Para el presente estudio se ha revisado la literatura médica desde 1962 hasta la actualidad, en diferentes idiomas, mediante búsquedas en Internet utilizando las bibliotecas Medline y Public-Med, junto con datos obtenidos de organizaciones oficiales. Se han utilizado además datos proporcionados por organizaciones pro-ayuda infantil, tanto estatales como privadas. Las publicaciones consideradas pertinentes se han ordenado y analizado ${ }^{19}$.

\section{Consideraciones generales y epidemiológicas}

El síndrome de maltrato o abuso infantil, también denominado en la literatura anglosajona como "Battered" o "Shaken Baby Syndrome", se puede definir como todo daño producido en un niño a consecuencia de una agresión directa o negligencia en su cuidado ${ }^{1}$. Aquí se incluyen los daños físicos, psicológicos y sociales, producidos en niños entre 0 y 18 años, por agresiones esporádicas o más frecuentemente reiteradas, tanto de sus progenitores como de familiares, conocidos o extraños, Dentro de esta amplia definición, se pueden distinguir 4 grupos diferentes: maltrato físico, agresión sexual, abandono o negligencia en su cuidado o atención médica y maltrato psicológico o emocional ${ }^{19}$. Sin embargo, existen formas características de ciertos países concretos, así tenemos prostitución infantil, mendicidad (niños de la calle), niños de la guerra o terrorismo, trabajos forzados, mutilación (sobretodo sexual en niñas), patología paterna de tipo psiquiátrico o drogadicción que afecta a los niños...

Es muy dificil establecer la incidencia y prevalencia del maltrato infantil. Dada la imposibilidad de establecer la causa exacta de las lesiones pediátricas, no es sorprendente que muchas sean consideradas caídas accidentales, 


\section{Incidencia de maitrato infantil en USA.}

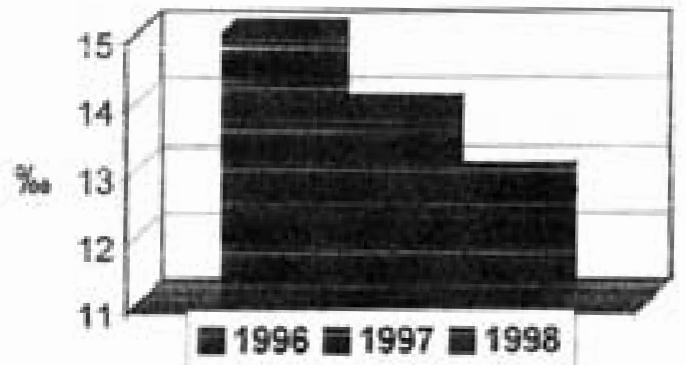

Figura 1. Incidencia de maltrato infantil en USA por cada 1000 niños $^{19}$.

encubriéndose un maltrato físico. La mayoría de los abusos sexuales, negligencias y maltratos psicológicos nunca se denuncian. Los datos existentes no siempre se publican de forma reglada en todos los países y regiones, haciendo aun más difícil conocer con exactitud su magnitud. Aun así vamos a intentar obtener una idea lo más exacta posible de la dimensión del problema.

Refiriéndonos a las estadísticas de los Estados Unidos, se estima que en 1998 hubo unos 900.000 niños maltratados, constituyendo una incidencia de 12,9 por cada 1000 niños $^{19}$. En la Figura 1 se ve que la cifra ha descendido levemente respecto a años anteriores (13,9\%o en 1997 y $15 \%$ en 1996$)^{18}$. De ellos, aproximadamente en el $18 \%$ se pone en peligro la vida del niño, muriendo un total de 1200 niños anualmente (Figura 2), el 1,5\%o de los niños maltratados de alguna forma ${ }^{17}$. En cuanto al tipo de maltrato, el 53,5\% sufre abandono, el 22,7\% maltrato físico, el $11,5 \%$ abuso sexual, el $6 \%$ maltrato psicológico y un $6 \%$ abandono de cuidados médicos ${ }^{19}$. La mayor incidencia se produce entre los más pequeños, siendo el 78\% de los maltratados menores de 3 años y el 38\% menores de 1 año ${ }^{25}$. Se da la circunstancia que los lactantes son más vulnerables a traumatismos craneoencefálicos con graves repercusiones, incluida la muerte. No existe diferencia significativa en cuanto al sexo, aunque algunos trabajos dicen que el maltrato físico es mayor en los niños, y el abuso sexual en las niñas entre 12 y 15 años $^{19}$.

Refiriéndonos ahora al autor del maltrato, la gran mayoría (77-87\%) está causado por alguno de los padres del niño, siendo un $11 \%$ por otros familiares o conocidos ${ }^{19}$. En la mitad de los casos existe un solo autor y una sola víctima. Los hombres suelen ser más propensos a maltrato físico y abuso sexual, mientras que las mujeres suelen ser culpables de abandono o negligencia.

\section{Consecuencias del maltrato}

Como ya hemos mencionado, existe una alta mortali-

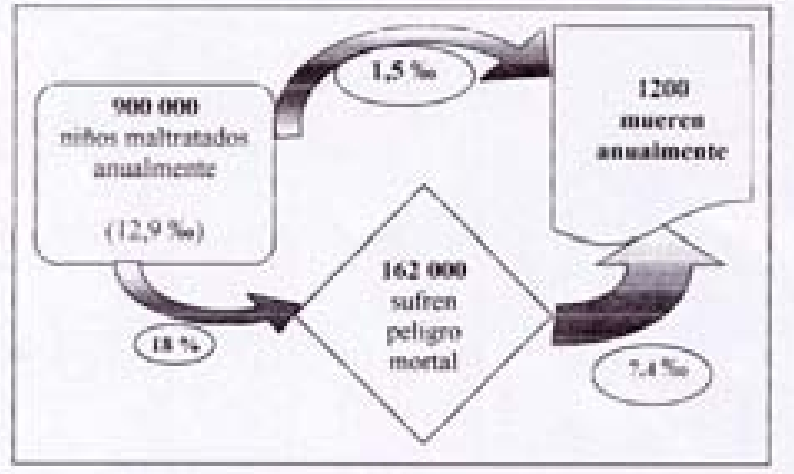

Figura 2. Incidencia de peligro vital y mortalidad en USA en $1998^{17}$.

dad, alcanzando hasta 15 niños por cada 10.000 maltratados (Figura 2). Se calcula que en Estados Unidos existen 1,6 muertes infantiles por cada 100.000 niños de la población general. La morbimortalidad ha ido aumentando con los años, pero presumiblemente por una mejor detección y registro, no realmente porque ahora tenga peores consecuencias que antes. La mortalidad es mayor entre los más pequeños, siendo el 77,5\% de todos los éxitus niños menores de 3 años ${ }^{25}$.

También existe una alta morbilidad. Se calcula que más del $10 \%$ de los niños con retraso mental y parálisis cerebral son debido a maltrato. Dentro de todas las posibles secuelas de un maltrato infantil, nos vamos a centrar en las secuelas neurológicas. En 1992 se analizaron en Estados Unidos las repercusiones del maltrato infantil ${ }^{19}$, encontrando que el $30 \%$ presentaban algún tipo de déficit cognitivo o de lenguaje, el $22 \%$ tenían trastornos de aprendizaje, el 50\% presentaban dificultad en la escuela, incluyendo déficit de atención y trastornos de conducta, el 50\% de los niños maltratados presentaban problemas emocionales y cerca del 14\% mostraban comportamientos autodestructivos. Otros estudios muestran una recuperación pobre tras maltratos físicos con traumatismo craneal severo, hallando un 65\% de niños con incapacidad moderada y un $15 \%$ con incapacidad grave (en la Escala de Resultados de Glasgow) ${ }^{4}$. Se ha visto que son factores de mal pronóstico neuropsicológico los niños menores de 10 años con traumatismos craneoencefálicos, especialmente en menores de 4 años, puntuación baja en la Escala de Coma de Glasgow al ingreso, ausencia de reactividad pupilar, amnesia postraumática que dure más de una semana, hallazgos radiológicos como edema cerebral difuso o lesión focal expansiva en la TC craneal y lesiones córtico-subcorticales y en sustancia blanca en la Resonancia Magnética ${ }^{3}$.

El tipo de maltrato que más secuelas neurológicas produce es el maltrato físico ${ }^{19}$. Dos tercios de los niños menores de 3 años maltratados físicamente sufren lesiones cerebrales. Además, el abandono puede ocasionar desnu- 
trición grave, que sobre todo en los primeros 6 meses de vida se asocia a secuelas neurológicas permanentes, potenciando las posibles lesiones de un maltrato físico ${ }^{24}$.

Las consecuencias del maltrato infantil no sólo se explican por las lesiones macroscópicas producidas en el sistema nervioso central. Existen trabajos que proponen que el trauma emocional del maltrato también altera la bioquímica cerebral. Así estos niños pueden tener alteraciones en las hormonas, como el cortisol o la norepinefrina, o bien puede estar alterado el número de sus receptores intracelulares ${ }^{16}$. Se crea entonces un estado de "hiperexcitabilidad" permanente, que les condiciona un estrés crónico. Esto puede alterar la respuesta a futuros estímulos estresantes, ya sea por exceso o por defecto. Se han encontrado cambios en las conexiones fronto-límbicas y alteraciones en los núcleos adrenérgicos del troncoencéfalo, incluyendo el "locus ceruleus" y los "núcleos del rafe".

Cuando un niño nace, su cerebro no está desarrollado y todas sus áreas no funcionan de una forma completa. Durante el crecimiento, el cerebro se desarrolla y madura. Es durante estos primeros momentos cuando el cerebro es más sensible y receptivo al exterior. Si el niño vive experiencias positivas, el cerebro se desarrolla sano y adquiere sus capacidades. Sin embargo, si existe una interrupción de los estímulos positivos o si, peor aún, nunca han existido, puede afectar profundamente al desarrollo funcional del cerebro del niño, con consecuencias irreversibles. Debido a esto, los niños menores de 5 años de edad son más vulnerables y presentan mayores secuelas a causa del maltrato ${ }^{24}$.

Desde 1990 se considera la lesión cerebral traumática un motivo de incapacidad, por la necesidad de educación especial de muchos de estos niños ${ }^{19}$.

Vamos a dividir las secuelas neurológicas en tres grupos, según sean consecuencia de un daño cerebral o craneal, daño en la columna o en la médula espinal y daño del sistema nervioso periférico. Habitualmente los daños del sistema nervioso periférico se incluyen dentro de las secuelas músculo esqueléticas. Así que nos vamos a centrar en los dos primeros grupos.

\section{Secuelas neurológicas por daño craneal o cerebral}

Vamos a dividir estas secuelas en cuatro grupos diferentes: secuelas motoras, visuales, del lenguaje o del aprendizaje y retraso mental o alteraciones de memoria.

\section{Secuelas motoras}

La lesión que más frecuentemente produce déficit motor son los hematomas subdurales agudos. Se forman por la rotura de venas puente entre la superficie cerebral y la dura durante los movimientos de aceleración y deceleración, muy frecuentes en estos casos (Baby Shaken
Syndrome), si bien estudios recientes sugieren que en la mayoría de estos niños también se produce daño por impacto, y no exclusivamente por la aceleración y deceleración ${ }^{5}$. Las localizaciones más frecuentes son las regiones de la convexidad cerebral e interhemisféricos, y se asocian en muchas ocasiones a contusiones cerebrales. En los estudios con TC tardíos se encuentran con similar frecuencia dos grupos diferentes, el primero con atenuación cerebral difusa (edad media en este grupo de 5 meses y 9 días) y el segundo con atenuación cerebral focal (edad media de 19 meses y 3 días). El daño en el parénquima es diferente, pero en ambos existe un mal pronóstico. Estos cambios en la TC se deben a procesos isquémicos en los capilares y venas corticales $^{8,9,20}$. Los daños producidos tras un hematoma subdural agudo pueden ser múltiples, incluyendo necrosis hemisférica (el más frecuente) ${ }^{23}$, infartos en los territorios de las arterias cerebral posterior y callosomarginal, infartos de "territorios frontera" como parieto-occipitales ${ }^{19}$, etc. Los cambios estructurales se aprecian a largo plazo en los estudios de Resonancia Magnética, pero para los cambios funcionales son más útiles los estudios nucleares (SPECT para la reserva vascular y PET para la función neuronal).

Los hematomas subdurales crónicos también pueden producir déficit motor. En el niño maltratado suelen ser bilaterales, y constituye un signo de sospecha de maltrato ${ }^{1,2}$. Los higromas subdurales y la hidrocefalia externa pueden deberse a la rotura de la aracnoides por un hematoma subdural previo, y son más frecuentes en el niño maltratado que en traumatismos craneoencefálicos accidentales ${ }^{3}$.

Otras causas menos frecuentes de déficit motor son las fracturas craneales y los hematomas epidurales, que son más frecuentes en caídas accidentales ${ }^{21}$. Debidos a los movimientos de aceleración puede producirse daño directo en las arterias, originando hemorragias subaracnoideas, que pueden originar isquemia cerebral por vasoespasmo, o pseudoaneurismas en las arterias cerebral media, carótida interna y coroideas posteriores ${ }^{7,22}$. Se han descrito aneurismas disecantes de la arteria vertebral y trombosis del seno lateral, aunque son poco frecuentes.

Las secuelas pueden deberse a la isquemia cortical ya descrita ${ }^{9}$, a lesión axonal difusa que daña las vías motoras en la sustancia blanca, o bien a daño en el troncoencéfalo debido a los desplazamientos y herniaciones producidas. Es preciso considerar que el cerebro de estos pacientes tiene mayor movilidad, que se ve incrementada por la desproporción cefálica y la pobre musculatura cervical ${ }^{19}$.

\section{Secuelas visuales}

El signo más frecuente del niño maltratado, y que cuando se ve suele orientarnos hacia un probable maltrato es la presencia de hemorragias retinianas ${ }^{2}$. Se producen en el $75-90 \%$ de los casos de maltrato físico, aunque tienen 
buen pronóstico, ya que se resuelven en casi todos los casos a los 4 meses. Son signos indicativos de maltrato, al igual que los hematomas subdurales bilaterales, las fracturas craneales que cruzan las suturas y las fracturas esqueléticas en diferentes estadios de evolución.

Pueden producirse daños en otras estructuras oculares, como las hemorragias vítreas, sin embargo, los déficits visuales son más frecuentemente debidos a daños cerebrales que oculares ${ }^{13}$.

Aunque la hemorragia retiniana en sí no produzca grandes secuelas, existe una correlación importante entre la extensión de dichas hemorragias con el grado de daño neurológico, debido a la coexistencia de lesiones cerebra$\operatorname{les}^{6}$.

\section{Secuelas del lenguaje y aprendizaje}

Las alteraciones del lenguaje se producen por daño estructural en el área de Broca, fascículo arcuato, área de Wernicke o gyrus angularis ${ }^{19}$. Se pueden manifestar como afasia o disfasia puramente motora, sensitiva, o más frecuentemente como una gran variedad de disfasias mixtas. Las disfasias sensitivas conllevan un retraso de aprendizaje, que a veces se confunde con retraso mental, pero que se debe al déficit sensorial que imposibilita la comprensión verbal $^{19}$. También puede existir un retraso de aprendizaje cuando se dañan otros órganos sensoriales, como el oído o la vista.

\section{Retraso mental y alteraciones de memoria}

El retraso mental postraumático es mucho mayor en los niños maltratados físicamente (hasta el 45\%) que en

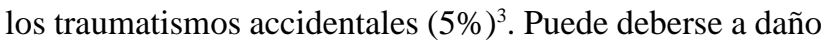
en lóbulos frontales, tálamo, etc, pero en niños sin ninguna otra secuela evidente, el maltrato por si mismo constituye una causa etiológica de retraso mental ${ }^{19}$.

Las alteraciones de memoria son las secuelas neuropsicológicas más frecuentes, y son causadas por lesión en lóbulos frontales, límbicos o temporo-mesiales ${ }^{19}$. Los déficits de memoria son mayores si el coma ha durado más de dos semanas, sobre todo para la fijación de la memoria a largo plazo. Es característico del maltrato infantil el daño en ambos lóbulos frontales, produciéndose secuelas de memoria y cierto grado de retraso mental.

\section{Secuelas neurológicas por daño medular o espinal}

Las lesiones espinales y medulares producidas en los niños maltratados son relativamente poco frecuentes, comparadas con las lesiones cerebrales. La parte más frecuentemente afectada es la columna cervical. Los niños presentan mucha menos proporción de lesiones óseas espinales que los adultos, debido a la gran laxitud ligamentosa, lo cual origina en muchos casos lesión medular sin ninguna lesión osteoligamentosa visible, el denominado síndrome de SCIWORA (Spinal Cord Injury Without Radiographic Abnormality). El mecanismo de lesión cervical suele ser por hiperflexión e hiperextensión extremas, que se ve favorecido en el lactante por la desproporción cráneo-corporal. Estudios anatomopatológicos muestran daños medulares, fundamentalmente axonales, desgarros en ligamentos paraespinales y hemorragias epidurales y subdurales ${ }^{14}$.

La mortalidad de las lesiones cervicales es muy alta, pudiendo quedar secuelas en los supervivientes, ya sean secciones medulares completas, con paraplejia o tetraplejia, según el nivel, o bien distintos grados de lesiones incompletas, muy frecuentemente asociados a trastornos esfinterianos ${ }^{14}$.

Otro lugar de asiento de lesiones espinales es la columna dorsal, o más frecuentemente la charnela cérvico-torácica. Es estos casos el traumatismo suele ser más importante y son frecuentes las lesiones asociadas, como fracturas de la escápula, indicando un traumatismo muy severo. También existe una alta mortalidad, y las secuelas son paraplejias o diferentes grados de paraparesias.

La lesiones tóraco-lumbares son menos frecuentes en los niños, y no suelen producir daño neurológico. El mecanismo más aceptado es la hiperflexión, produciéndose distintos tipos de lesiones, por lo general sin grandes secuelas $^{19}$.

\section{Rehabilitación}

Esta parte del tratamiento es esencial, y debe iniciarse muy precozmente. En muchas ocasiones las secuelas permanentes dependerán de la demora o la falta del tratamiento rehabilitador. En general, la recuperación de un traumatismo no accidental es peor que la de un accidente ${ }^{19}$. Los objetivos del tratamiento del traumatismo craneoencefálico pediátrico son muy claros, e incluyen asistencia inmediata y en el hospital, rehabilitación y educación de las habilidades perdidas y programas de reinserción en la comunidad, en el ambiente previo ${ }^{12}$. En la mayoría de las ocasiones éste es un proceso muy lento ${ }^{11}$.

Podemos dividir la rehabilitación de estos niños en dos apartados: rehabilitación física de los déficits neurológicos (generalmente motores), y rehabilitación de funciones cognitivas, incluyendo en este apartado la recuperación emocional. Sin embargo, ambas partes se realizan de forma conjunta, ya que una va a depender de la otra.

Para la rehabilitación de secuelas motoras se utilizan las mismas técnicas que en otros pacientes, cinesiterapia lo más precoz posible, primero pasiva, activa-asistida y finalmente activa. Se utilizarán prótesis y ortesis cuando sean necesarias. En casos de espasticidad es preciso la utiliza- 
ción precoz de fármacos o incluso tratamientos quirúrgicos disponibles, para obtener la mejor recuperación motora posible.

En lactantes es fundamental la estimulación continua y progresiva. A ello ha de acompañarse un gran refuerzo emocional, ya que es fundamental para el desarrollo neuronal. Entrando ya en la función cognitiva, estudios recientes sugieren que la recuperación de habilidades de cálculo, lectura y escritura es máxima en los 6 primeros meses, siendo muy pobre posteriormente ${ }^{10}$. Estos niños suele estar muy irritables y tener muy baja autoestima en los primeros momentos. Hay que potenciar sus habilidades enseñando estrategias nuevas que ayuden al niño a superar los déficits y posibles dificultades que puedan encontrar ${ }^{19}$. Este paso es fundamental, y ayudará también a la recuperación emocional, haciendo que los niños sean autosuficientes y potenciando su reintegración escolar y social. Muchos niños necesitarán programas de educación especial. Para todo ello existe un gran armamento, incluyendo programas asistidos por ordenador ${ }^{19}$.

Para evaluar de forma objetiva los progresos realizados, podemos estudiar el estado cerebral con pruebas de neuroimagen funcionales, como el SPECT, RM funcional y el PET. Existe una muy buena correlación entre la recuperación funcional y la mejoría en dichos estudios cerebrales ${ }^{15}$.

En todo este proceso debe existir una buena coordinación entre distintos especialistas: médico rehabilitador, pediatra, neurocirujano, neurólogo... El asistente social es fundamental, en colaboración con los programas para la protección del menor, ya que es imprescindible proporcionar el mejor ambiente familiar posible.

\section{Bibliografía}

1. Caffey, J.: On the theory and the practice of shaking infant: its potential residual effects of permanent brain damage and mental retardation. Am J Dis Child. 1972; 124: 161-169.

2. Caffey, J.: The whiplash shaken infant syndrome: Manual shaking by the extremities with whiplash-induced intracranial and intraocular bleedings, linked with residual permanent brain damage and mental retardation. Pediatrics. 1974; 54: 396-403.

3. Cooper, P., Golfinos, J.: Head Injury. Fourth Edition. McGraw-Hill. 2000.

4. Duhaime, A.C., Christian, C., Moss, E., et al.: Long term outcome in infants with the shaking-impact syndrome. Pediatr Neurosurg. 1996; 24: 292-298.

5. Duhaime, A., Christian, C., Rorke, L., et al.: Nonaccidental head injury in infant- The "shaken-baby syndrome ." N Engl J Med. 1998; 338: 1822-1829.

6. Duhaime, A., Gennarelli, T., Thibault, L., et al.: The shaken baby syndrome: A clinical, pathological, and biome- chanical study. J Neurosurg.1987; 66: 409-415.

7. Dujovny, M., Laha, R.K., DeCastro, S., Briani, S.: Post-traumatic middle cerebral artery thrombosis. J of trauma. 1980; 19: 775-779.

8. Dujovny, M., Wackenhut, N., Kossovsky, N., Gómez, C.W., Laha, R.K., Leff, L., Nelson, D.: Minimal vascular occlusive force. J Neurosurg. 1979; 51: 662-688.

9. Dujovny, M., Wakenhut, N., Kossovsky, N., Leff, L., Gómez, C., Nelson, D.: Biomechanics of vascular occlusion in neurosurgery. Acta Neurol Latinoamer. 1980; 26: 12-127.

10. Ewing-Cobbs, L., Fletcher, J., Levin, Iovino, I., Miner, M.: Academic achievement and academic placement following traumatic brain injury in children and adolescents: a two-year longitudinal study. J Clin Exp Neuropsychol. 1998; 20: 769-781.

11. Hostler, S.: Pediatric family-centered rehabilitation. J Head Trauma Rehabil. 1999; 14: 384-393.

12. James, H.: Pediatric head injury: what is unique and different. Acta Neurochir (Wien). Suppl. 1999; 73: 85-88.

13. Kivlin, J.D., Simons, K.B., Lazoritz, S., Ruttum, M.S.: Shaken baby syndrome. Ophtalmology. 2000; 107: 12461254.

14. Kleinman, P.K., Shelton, Y.A.: Hangman's fracture in an abused infant: imaging features. Pediatr Radiol. 1997; 27: 776-777.

15. Laatsch, L., Jobe, T., Sychra, J., Lin, Q., Blend, M.: Impact of cognitive rehabilitation therapy on neuropsychological impairments as measured by brain perfusion SPECT: a longitudinal study. Brain Inj. 1997; 11: 851-863.

16. Macias, R., Chagoya, L.: Forms of child abuse in the family. Gac Med Mex. 1975; 109: 235-244.

17. Murphy, S.L.: Deaths: Final data for 1998. Natl Vital Stat Rep 2000; 48: 1-105.

18. Pedle, N., Wang, C.T., Díaz, J., Ried, R.: Current Trends in Child Abuse Reporting and Fatalities: The Results of the 2000 Annual Fifty State Survey. National Center on Child Abuse Prevention Research, a program of Prevent Child Abuse America. Chicago 2002. www.preventchildabuse.orq

19. Pérez-Arjona, E., Dujovny, M., Vinas, F., Park, H., Lizárraga, S., Park, T., Díaz, F.G.: CNS child abuse: epidemiology and prevention. Neurol Res. 2002; 24: 29-40.

20. Rozario, R., Dujovny, M., Wackenhut, N., Worly, P., Semba, A.: Temporary microvascular occluder. Neurosurgery. 1981; 8: 454-457.

21. Shugerman, R.P., Paez, A., Grossman, D.C., Feldman, K.W., Grady, M.S.: Epidural hemorrhage: is it abuse? Pediatrics. 1996; 97: 664-668.

22. Vinas, F.C., López, F., Dujovny, M.: Microsurgical anatomy of the posterior choroidal arteries. Neurol Res. 1995; 17: 334-344.

23. Zimmerman, R.A., Bilaniuk, L.T., Bruce, D., Schut, L., Uzzell, B., Goldberg, H.I.: Computed Tomography of craniocerebral injury in the abused child. Radiology. 1979; 130: 
687-690.

24. www.aacap.org/publications/factsfam/chlabus.htm. Child Abuse-The Hidden Bruises. American Academy of Child\&Adolescent Psychiatry. 1998.

25. www.childhelpusa.org . Child Abuse in America. Childhelp USA Statistics 2000

\section{Comentario al trabajo Secuelas neurológicas del mal- trato infantil. Revisión bibliográfica de Giménez-Pando y cols.}

Esta revisión sobre las secuelas del maltrato infantil de Giménez-Pando y cols. nos parece un interesante y relevante artículo, tanto por la recopilación reciente de datos, como por su enfoque en el tratamiento y rehabilitación de las secuelas neurológicas del niño maltratado. El tema, siempre actual, representa un desafío diagnóstico y rehabilitador continuo, y constituye un critico capítulo epidemiológico y social.

Insistiendo en la excelente revisión de los autores, creemos importante recordar tres factores diagnósticos: El primero es la muy probable subestimación del maltrato infantil tanto en la clínica diaria, como de forma genérica en los datos epidemiológicos, enfatizando la necesidad de pensar siempre en esta posibilidad diagnóstica.

El segundo es resaltar que la presentación clínica del maltrato infantil es diferente a la del traumatismo por accidente. Mientras que en el último son frecuentes los hematomas faciales y de cuero cabelludo, en el primero se observan comúnmente irritabilidad, nutrición deficiente, letargia, cambios en el estado mental y crisis convulsivas, unido todo ello a una historia clínica en la que no cuadra el mecanismo y/o la gravedad del trauma.
Giménez-Pando, J.; Perez-Arjona, E.; Dujovny, M.; Díaz, F.G.: Secuelas neurológicas del maltrato infantil. Revisión bibliográfica. Neurocirugía 2007; 18: 95-100.

Corresponedcia postal: Jorge Gimenez Pando. Calle Cardenal Ilundain $n^{\circ}$ 24, esc 4, $2^{\circ}$ C Sevilla. 41013. España.

El tercero es que aunque el maltrato infantil se puede hallar en cualquier entorno sociofamiliar, se consideran factores importantes de riesgo un pobre nivel de formación de la madre, un bajo status socioeconómico, la corta edad de los padres, una historia de abuso infantil en la familia, y factores estresantes recientes en el entorno de los padres ${ }^{1}$.

Dado que en más del $50 \%$ de los casos de esta compleja patología se halla presente el traumatismo craneal, y/o cervical y/o facial, es bueno insistir en la fundamental necesidad de nuestra colaboración como personas y neurocirujanos en la defensa de nuestros niños.

\section{Bibliografía}

1. Raja, A.L., Husain, E., Guerrero, C.A., Moseley, T., Adada, B.: Pediatric Nonaccidental Trauma. Contemporary Neurosurgery 2005; Vol. 27: 1-6.

F.J.Cordobés Madrid. 